\title{
Clinico-Hematological Profile of Patients with B-Chronic Lymphoid Leukemia in Pakistan
}

\author{
Rozina Zeeshan, Sadia Sultan*, Syed Muhammad Irfan, Jamaludin Kakar, \\ Muhammad Asif Hameed
}

\begin{abstract}
Background: Chronic lymphoid leukemia (CLL) is not an uncommon hematological malignancy which primarily affects elderly individuals. It is more common in developed world than in developing countries. The rational of this study was to determine the clinico-hematological profile in Pakistan. Materials and Methods: In this prospective cross sectional study, sixty patients with CLL were enrolled from January 2011 to June 2013. Data were analyzed with SPSS version 21. Results: The mean age was 59.0 \pm 9.2 years (range 40-82) and the male to female ratio was 2.1:1. Peak age group was 60-70 years $(38.3 \%)$ and $18.3 \%$ were under 50 years old. Major complaints were weakness $(\mathbf{5 1 . 7 \%})$, fever $(\mathbf{1 8 . 3 \%})$ and abdominal discomfort $(\mathbf{1 3 . 3 \%})$. Main clinical findings were splenomegaly $(\mathbf{4 6 . 6 \% )}$, lymphadenopathy $(36.6 \%)$ and pallor $(26.7 \%)$. Some $16.7 \%$ were diagnosed incidentally. The mean hemoglobin was $10.8 \pm 2.4 \mathrm{~g} / \mathrm{dl}$, with a total leukocyte count of $91.5 \pm 87.8 \times 10^{9} / 1$ and platelets 197.8 $\pm 103.2 \times 10 \% /$. Anemia and thrombocytopenia were seen in $26.7 \%$ and $21.7 \%$ of cases, respectively. High LDH and hyperuricemia were detected in $15 \%$ each and elevated serum creatinine was seen in $11.6 \%$. According to Rai staging $11.6 \%$ were in stage $0,13.3 \%$ stage $1,26.7 \%$ each for stage II and stage III while $21.7 \%$ patients were in stage IV. Conclusions: CLL in our patients in Pakistan, unlike in the West, is seen in a relatively young population with male predominance. Primarily disease is of $\mathrm{B}$ - cell origin and about $2 / 3$ of the patients present at advanced stage.
\end{abstract}

Keywords: Chronic lymphoid leukemia - clinico-hematological - B-CLL - Pakistan

Asian Pac J Cancer Prev, 16 (2), 793-796

\section{Introduction}

Chronic lymphoid leukemia (CLL) is a hematolymphoid malignancy characterized by a clonal proliferation and accumulation of nonfunctional and ineffectual small lymphocytes in the blood, bone marrow and lymphoid tissues (Khan et al., 2014; Kermani et al., 2007). It is the commonest adult leukemia in the western world, with the yearly incidence of 3-5 per 100,000 people (Vroblova et al., 2009; Maurer and Hallek, 2013).

Elderly individuals are mainly affected, with a median age of 70 years at the diagnosis and male preponderance (Redaelli et al., 2004). Chronic lymphoid leukemia accounts for approximately 22-30\% of all leukemia's and $25 \%$ of Non Hodgkin's lymphoma (Maurer and Hallek, 2013; Redaelli et al., 2004). However, CLL is very rare in far Eastern countries but incidence is high in Australia, USA, Ireland and Italy (Redaelli et al., 2004). CLL in our area is reported as least frequent hematological malignancy, accountable in $9.7 \%$ amongst all the leukaemias (Dodhy et al., 2011).

The prognosis and clinical spectrum of chronic lymphoid leukemia's patients is heterogeneous. Some patient experience rapid disease progressions while others existing for a decade without any treatment (D'Arena et al., 2007; Gribben, 2008; Qi et al., 2009). Patients may present with localized or generalized lymphadenopathy, hepatosplenomegaly, cytopenias or constitutional symptoms (Hamblin, 2007). However a number of patients are asymptomatic at the time of diagnosis and are only identified by the incidental finding of lymphocytosis on routine investigation (Sriphatphiriyakun and Auewarakul, 2005).

Several clinical and laboratory factors have been well established, which have a direct impact on the disease course and its outcome. Certain factors are incorporated in the conventional staging systems (Rai and Binet) which are still widely applicable and effective to dictate the overall survival (Mozaheb et al., 2012). But these routinely used staging systems do not discriminate precisely the probable disease pace at the initial diagnosis.

This study mainly explores the basic clinical and laboratory parameters of newly diagnosed chronic lymphoid leukemia patients and the stage of disease. 


\section{Materials and Methods}

This prospective cross sectional study, extended from January 2011 to June 2013. 60 patients with chronic lymphoid leukemia were enrolled in the study. All patients were registered in hematology clinic where there record was maintained including history, physical examination and follow ups. An informed consent was obtained from all the participating patients.

Patients with other acute or chronic lymphoproliferative disorders, post chemotherapy/radiotherapy, having non hematopoietic malignancy or with relapsed/ refractory CLL were excluded.

Hematological parameters included hemoglobin, WBC count, platelets and absolute lymphocytic count were determined by Cell Dyne (Architect Abott, USA). Immunophenotyping was done by flow cytometry or immunohistochemistry to diagnose chronic lymphoid leukemia (CD19, CD20, CD5 and CD23 positivity and FMC7, cyclin D1 and BCL2 to be negative for diagnosis of B-CLL). Serum creatinine, lactate dehydrogenase (LDH) and serum uric acid were detected by HITACHI 912 (Japan) by photometric assay. Bone marrow aspirate and trephine biopsy specimen were taken through Jamshidi needle.

The ethical endorsement of the study was granted by research and ethical committee LNH taken prior to the study.

\section{Data analysis}

Data was compiled and analyzed using SPSS version 21 . The results were expressed as mean \pm SD for quantitative variables and qualitative variables are presented as frequency \& percentages.

\section{Results}

During the study period, 66 patients were diagnosed as chronic lymphoid leukemia. Immunophenotyping revealed B-CLL in 60 (91\%) patients while remaining 6 patients (9\%) were of T-CLL phenotype. Results presented below are on B- CLL patients.

Out of sixty patients 42 were males (70\%) and 18 were females (30\%) with male to female ratio of 2.1:1. The mean age at presentation was 59.0 \pm 9.2 (range 40-82) years. Mean age of male and female were 58.2 \pm 8.97 and $60.0 \pm 10.01$ respectively. Most frequent age group was $60-70$ years $(38.3 \%)$ while 11 patients $(18.3 \%)$ were $<50$ years of age.

The presenting complains were weakness in 31 patients $(51.7 \%)$, followed by fever in 11 patients $(18.3 \%)$ and abdominal discomfort in 8 patients (13.3\%). The $\mathrm{r}$ emaining $16.7 \%$ were asymptomatic at presentation. Physical examination revealed splenomegaly as predominant finding detected in 28 patients (46.6\%), followed by lymphadenopathy in 22 patients (36.6\%) and pallor was seen in $16(26.7 \%)$ patients.

The mean hemoglobin level was $10.8 \pm 2.4 \mathrm{~g} /$ dl (range 2.7-15.8) while mean TLC count was $91.5 \pm 87.8 \times 10^{9} / 1$ (range 16-448). The mean platelets count were $197.8 \pm 103.2 \times 10^{9} / 1$ (range $71-560$ ) and absolute lymphocytic count was $91.88 \pm 69.43 \times 10^{9} / \mathrm{L}$ (range 14 -

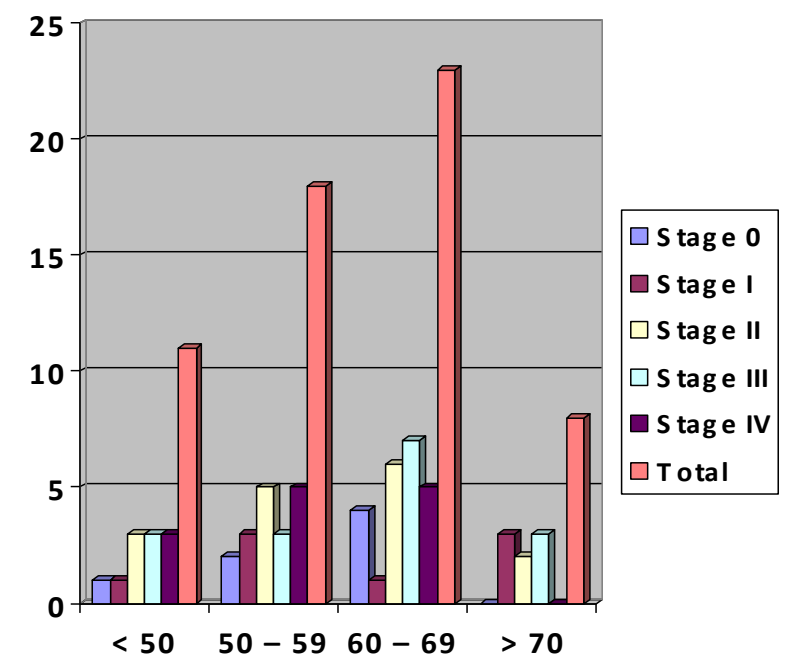

Figure 1. Age Distribution of Pakistani CLL Patients

Table 1. Comparison with Other Regional Studies

\begin{tabular}{lcccccc}
\hline Clinical Features & Present Study 2014 & China 2008 & Thailand 2005 & Nigeria 2007 & India 2007 & Central Africans 2004 \\
\hline Peak age group & $60-70$ & NA & $60-80$ & $51-60$ & $60-70$ & $60-64$ \\
Mean Age & $59 \pm 9.2$ & 60 & $62.8 \%$ & 56 & 61 & 56.8 \\
Male : Female & $2.1: 1$ & $2.1: 1$ & $2: 1$ & $1: 3$ & $4.6: 1$ & 1.1 \\
Weakness & $51.7 \%$ & $54.8 \%$ & $39 \%$ & NA & $22 \%$ & NA \\
Fever & $18.3 \%$ & NA & $21 \%$ & NA & NA & NA \\
Abdominal discomfort & $13.3 \%$ & NA & NA & $58.3 \%$ & NA & NA \\
Lymphadenopathy & $36.6 \%$ & $54.8 \%$ & $60 \%$ & $91.7 \%$ & $55 \%$ & $33.3 \%$ \\
Splenomegaly & $46.6 \%$ & NA & $64 \%$ & $50 \%$ & $66 \%$ & $68 \%$ \\
Pallor & $26.7 \%$ & NA & $54 \%$ & $58.3 \%$ & $27 \%$ & $34.7 \%$ \\
Thrombocytopenia & $21.7 \%$ & NA & $18 \%$ & NA & $18 \%$ & NA \\
\hline
\end{tabular}

Table 2. Distribution of CLL Patients According to Age and Rai Disease Stages

\begin{tabular}{lcccccc}
\hline Age Group & Stage 0 & Stage I & Stage II & Stage III & Stage IV & Total \\
\hline$<50$ & 1 & 1 & 3 & 3 & 3 & 11 \\
$50-59$ & 2 & 3 & 5 & 3 & 5 & 18 \\
$60-69$ & 4 & 1 & 6 & 7 & 5 & 23 \\
$>70$ & 0 & 3 & 2 & 3 & 0 & 8 \\
Total & 7 & 8 & 16 & 16 & 13 & 60 \\
Percent & $11.6 \%$ & $13.3 \%$ & $26.7 \%$ & $26.7 \%$ & $21.7 \%$ & $100 \%$ \\
\hline
\end{tabular}


380). Hyperuricemia and high LDH were detected in $15 \%$ of each with the mean level of $5.59 \pm 2.47 \mathrm{mg} / \mathrm{dl}$ and $512 \pm 232.61 \mathrm{u} / \mathrm{l}$ respectively. Elevated serum creatinine was seen in $11.6 \%$ with the mean $1.29 \pm 0.56 \mathrm{mg} / \mathrm{dl}$. Bone marrow biopsy showed involvement in virtually all patients with different spectrum of infiltrations.

According to Rai staging $11.6 \%$ (7 patients) were in stage $0,13.3 \%$ (8 patients) had stage $1,26.7 \%$ (16 patients) each for stage II and stage III while $21.7 \%$ (13 patients) were in stage $1 \mathrm{~V}$ respectively.

\section{Discussion}

CLL is an indolent malignancy of mature small lymphocytes of unknown etiology (Li ZJ et al., 2008). It usually originates from B lymphocytes that exhibit heterogeneousity at molecular level (Khan et al., 2014). This hematological disease is not uncommon in Pakistan, but due to insufficient diagnostic facilities and virtually lack of health education they are diagnosed at late stages when treatment is either impractical or very complex (Idris, et al, 2004).

In the USA, approximate $95 \%$ of CLL cases are of B-cell phenotype, whilst in Asia, T-cell CLL is also not uncommon. In our hands, T- CLL is accountable in $9 \%$ Pakistani patients. The difference in phenotype between Asian and western population is unclear (Kawamata et al., 2013). One probable explanation may be the difference between two ethnic groups based on genetic and molecular levels (Kawamata et al., 2013). Another explanation could be environmental factor with underlying infective etiology leading to chronic persistent stimulation (Kawamata et al., 2013). The present study illustrated that B-CLL is the predominant phenotype in Pakistani patients which is in consensus with other regional studies (Agrawal et al., 2007).

It is consistently found that CLL is the disease of older age (Mukiibi et al., 2004). Most of the patients in our study presented in the 6th decade of life. Similarly reported by Gogia et al from India, the median age of CLL patients was 59 years in a large series (Gogia et al., 2011). When compared with earlier reports, our results are in concurrence with local studies published from Lahore (Ehsan et al., 2013; Khalid et al., 1997). CLL, affected a much younger population in our hands, although the male gender dominance was similar to that reported in international and regional studies (Khan et al., 2014; Maurer and Hallek, 2013).

Our patients with chronic lymphoid leukemia often presented with constitutional symptoms (B symptoms) including weight loss and fever (due to the catabolic state), seen in $51.7 \%$ and $18.3 \%$ respectively. The Thai and Indian CLL patients were also found to have B symptoms accountable, in $33 \%$ and $60 \%$ respectively. (Sriphatphiriyakun and Auewarakul, 2005; Agrawal et al., 2007). The presence of $B$ symptoms is a marker for more advanced disease with systemic, rather than merely local involvement and is definitive indication for treatment.

Anemia is a serious and frequent manifestation in chronic lymphoid malignancy, associated with poorer prognosis, advanced stage and increased morbidity. Its prevalence has been reported in around $26-43 \%$ of CLL patients (Ghosh et al., 2013). Multifactorial pathogenesis contributes in the development of anemia which includes anemia of chronic disease, nutritional deficiencies, immune mediated or bone marrow infiltrations. In the present study, relatively high prevalence $(26.7 \%)$ was detected in comparison with one prior $(15.1 \%)$ regional study (Dodhy et al., 2011). Salawuet et al from Nigeria reported very high prevalence of anemia (74.4\%). However in our patients prevalence was more or less similar to studies reported from India (27\%) and Central Africa (34.7\%) (Mukiibi et al., 2004; Agarwal et al., 2007).

The mean total leukocytic counts and absolute lymphocytes in our series are $91.5 \times 10^{9} / 1$ and $91.8 \times 10^{9} / \mathrm{L}$ respectively. In disparity to our results one study from India, revealed relatively low median white blood cells and absolute lymphocytic count at presentation, 70.6x10 10 and $51.4 \times 10^{9} / 1$ respectively (Agrawal et al., 2007). This variation represent high disease burden in our patients could have been due to the late presentation as many patients in our country avoid medical consultation and ignore general health practices.

Thrombocytopenia at presentation has always been found to be a bad prognostic indicator with stage IV disease. In our series, it has been detected in $21.7 \%$ patients. In accordance to our findings, prior study from Thailand reported $18 \%$ patients had low platelets with a trend towards decreased survival for stage IV disease with thrombocytopenia (Sriphatphiriyakun and Auewarakul, 2005).

High serum creatinine may signify advanced stage of disease, seen in $11.6 \%$ of our patients (Rafiq et al., 2014). Rafiq N, et al reported serum creatinine of $4.6 \pm 1.4$ $\mathrm{mg} / \mathrm{dl}$ compared to our findings of $1.29 \pm 0.56 \mathrm{mg} / \mathrm{dl}$. This markedly high creatinine could be the reflection of advanced stage, as the mean platelets count was reported as $46.0 \pm 70.2 \times 10^{9} / 1$ and mean hemoglobin was $7.4 \pm 1.6 \mathrm{~g} /$ dL in their study (Rafiq et al., 2014).

Though bone marrow examination is not required to establish the diagnosis in general, but it will discriminate between good and bad risk CLL. Moreover the bone marrow examination is particularly important to differentiate between the causes of cytopenia and patterns of lymphoid infiltrate will help to differentiate with other lymphoproliferative disorders. Diffuse bone marrow involvement is the sign of bad prognosis and indicative of progressive advanced disease. Majority of our patient's revealed diffuse involvement of trephine biopsy, which is in accordance with Chinese study which revealed $72.2 \%$, had diffuse involvement of trephine (Li et al., 2008).

The Rai staging system is useful to delineate the disease extent and to predict the likelihood of survival. Approximately $80 \%$ of newly diagnosed patients belonged to low risk group in developed countries (Sagatys and Zhang, 2011; Tjønnfjord et al., 2012). Compared with data from developed countries, majority of our patients $(48.4 \%)$ presented in an advanced stages of disease. Similarly patients from Nigeria and India were also presented in late stages (Omoti et al., 2007; Gogia et al., 2011). This difference may be accredited to the aggressive disease course along with concomitant factor of delay in 
Rozina Zeeshan et al

seeking medical attention. Generally low risk group (Rai stage 0 ) have a median survival $>10$ years (Sagatys and Zhang, 2011).

The limitations of the study need to be mentioned; one is small sample size and secondly some novel informative markers including molecular testing, mutational status, ZAP-70 and karyotyping are not presented here.

In conclusion, the study reveals that CLL is not uncommon in Pakistan. Predominantly disease is of B cell origin. Presenting features are similar as reported in literature. But unlike west most of the patients presented in advanced stages with unfavorable prognostic factors; predominant male gender, constitutional symptoms, stage III/IV disease, high LDH and bone marrow diffuse infiltrates. Prospective studies should be pursued on large patient series to investigate further disease spectrum and should incorporate novel prognostic molecular testing.

\section{Acknowledgements}

The authors are grateful to the patients who have participated in this study. We thank staff of the Hematology Division of Liaquat National Hospital, for their excellent support.

\section{References}

Agrawal N, Naithani R, Mahapatra M, et al (2007). Chronic lymphocytic leukemia in India - a clinico-haematological profile. Hematology, 12, 229-33.

D’Arena G, Tarnani M, Rumi C, et al (2007). Prognostic significance of combined analysis of ZAP-70 and CD38 in chronic lymphocytic leukemia. Am J Hematol, 82, 787-91.

Dodhy MA, Zafar H, Aslam W (2011). Chronic Lymphocytic Leukemia: an Experience of a decade at a Tertiary Care Hospital. Ann Pak Inst Med Sci, 7, 196-9.

Ehsan A, Lone A, Khan MA (2013). Autoimmune complications in chronic lymphocytic leukemia: a single center experience. Biomedica, 29, 37-41.

Ghosh J, Singh RK, Saxena R, et al (2013). Prevalence and aetiology of anaemia in lymphoid malignancies. Natl Med J India, 26, 79-81.

Gogia, A. Sharma, V. Raina, et al (2011). Clinico-hematological characteristics and outcome assessment of patients with chronic lymphocytic leukemia. J Clin Oncol, 29, 2011.

Gribben JG (2008). Molecular profiling in CLL. Hematology Am Soc Hematol Educ Program. 2008, 444-9.

Hamblin TJ (2007). Prognostic markers in chronic lymphocytic leukaemia. Best Pract Res Clin Haematol, 20, 455-68.

Idris M, Shah SH, Fareed J, Gul N (2004). An experience with sixty cases of haematological malignancies; a clinico haematological correlation. J Ayub Med Coll Abbottabad, 16, 51-4.

Kawamata N, Moreilhon C, Saitoh T, et al (2013). Genetic differences between Asian and Caucasian chronic lymphocytic leukemia. Int J Oncol, 43, 561-5.

Kermani IA, Dehdilani M, Dolatkhah R (2007). Chronic lymphocytic leukemia in the recent 10 years and treatment effects of fludarabin. Asian Pac J Cancer Prev, 8, 367-71.

Khan M, Saif A, Sandler S, Mirrakhimov AE (2014). Idelalisib for the treatment of chronic lymphocytic leukemia. ISRN Oncol, 2014, 931858.

KhalidA,Zahid M,RehmanA, et al (1997).Clinicoepidemiological features of adult leukemias in Pakistan. Pak Med Assoc, 47,
119-22.

Li ZJ, Qiu LG, Wu T, et al (2008). The clinical and laboratory features of 263 cases of chronic lymphocytic leukemia. Zhonghua Xиe Ye Xue Za Zhi, 29, 300-3.

Maurer C, Hallek M (2013). Chronic lymphocytic leukemia. Dtsch Med Wochenschr, 138, 2153-66.

Mozaheb Z, Hasanzadeh NazarAbadi MH, Aghaee MA (2012). Chronic lymphocytic leukemia and prognostic factors. Asian Pac J Cancer Prev, 13, 3009-13.

Mukiibi JM, Paul B, Nyirenda CM, et al (2004). Chronic lymphocytic leukaemia (CLL) in Central Africans. Cent Afr J Med, 50, 111-5.

Omoti CE, Awodu OA, Bazuaye GN (2007).Chronic lymphoid leukaemia: clinico-haematological correlation and outcome in a single institution in Niger Delta region of Nigeria. Int $J$ Lab Hematol, 29, 426-32.

Qi RJ, Zhang PH, Qiu LG, et al (2009). Clinical significance of ZAP-70 protein expression in chronic lymphocytic leukemia/ small lymphocytic lymphoma. Zhonghua Bing Li Xue Za Zhi, 38, 329-32.

Rafiq N, Iqbal T, Shahid M, Muhammad F (2014). Hematological and biochemical parameters in Pakistani chronic lymphoblastic leukemia patients. Pak J Life Soc Sci, 12, 16-19.

Redaelli A, Laskin BL, Stephens JM, Botteman MF, Pashos CL (2004). The clinical and epidemiological burden of chronic lymphocytic leukaemia. Eur J Cancer Care Engl, 13, 279-87.

Sagatys EM, Zhang L (2012). Clinical and laboratory prognostic indicators in chronic lymphocytic leukemia. Cancer Control, 19, 18-25.

Salawu L, Bolarinwa RA, Durosinmi MA (2010). Chronic lymphocytic leukaemia: a-twenty-years experience and problems in Ile-Ife, South-Western Nigeria. Afr Health Sci, 10, 187-92.

Sriphatphiriyakun T, Auewarakul CU (2005). Clinical presentation and outcome of Thai patients with chronic lymphocytic leukemia: retrospective analysis of 184 cases. Asian Pac J Allergy Immunol, 23, 197-203.

Tjønnfjord GE, Ly BE, Johannesen TB, et al (2012). Chronic lymphocytic leukaemia in Norway--incidence and prognostic markers at diagnosis. Tidsskr Nor Laegeforen, 132, 2056-9.

Vroblova V, Smolej L, Vrbacky F, et al (2009). Biological prognostic markers in chronic lymphocytic leukemia. Acta Medica Hradec Kralove, 52, 3-8. 